\title{
The Second Lepton Family
}

\author{
Klaus Winter, CERN
}

The Nobel Prize for Physics for 1988 was awarded to L. Lederman, M. Schwartz and J. Steinberger for work on neutrinos in the early 1960s.

In a letter [1] addressed to the "dear radioactive ladies and gentlemen", written in December 1930, Wolfgang Pauli proposed, as a "desperate remedy" to save the principle of conservation of energy in beta-decay, the idea of the neutrino, a neutral particle of spin $1 / 2$ and with a mass not larger than 0.01 proton mass. "The continuous beta-spectrum [2] would then become understandable by the assumption that in beta-decay a neutrino is emitted together with the electron, in such a way that the sum of the energies of the neutrino and electron is constant."

Pauli did not specify at that time whether the neutrino was to be ejected or created. In his famous paper "An attempt of a theory of beta-decay" [3] E. Fermi used the neutrino concept of Pauli together with the concept of the nucleon of Heisenberg. He assumed that in beta-decay a pair comprising an electron and a neutrino is created, analogous to photons in transitions between nuclear states. This theory accounted successfully for the observed continuous electron spectrum in beta-decay.

Detection of the recoils of nuclei produced in beta-decay in coincidence with the electron had already given reality to the neutrino. Cowan and Reines [4] first detected antineutrinos from fission fragments by observing the inverse betadecay of protons.

The universal V-A theory of Feynman and Gell-Mann [5] described successfully all weak decays by a local currentcurrent interaction. According to this theory, the cross-sections of neutrino reactions would increase with energy and lead to a unitarity catastrophe at the so-called Fermi scale, $G_{F}^{-1 / 2}=300 \mathrm{GeV}$. An experimental investigation of the behaviour of the weak interaction at high energy therefore became a central question.

Following Feynman and Gell-Mann the divergency inherent in a point-like coupling of the weak current could be avoided by postulating that the currentcurrent coupling is transmitted by the exchange of massive charged bosons $W^{ \pm}$. However, there was already a problem. Feinberg [6] asked: "Why does

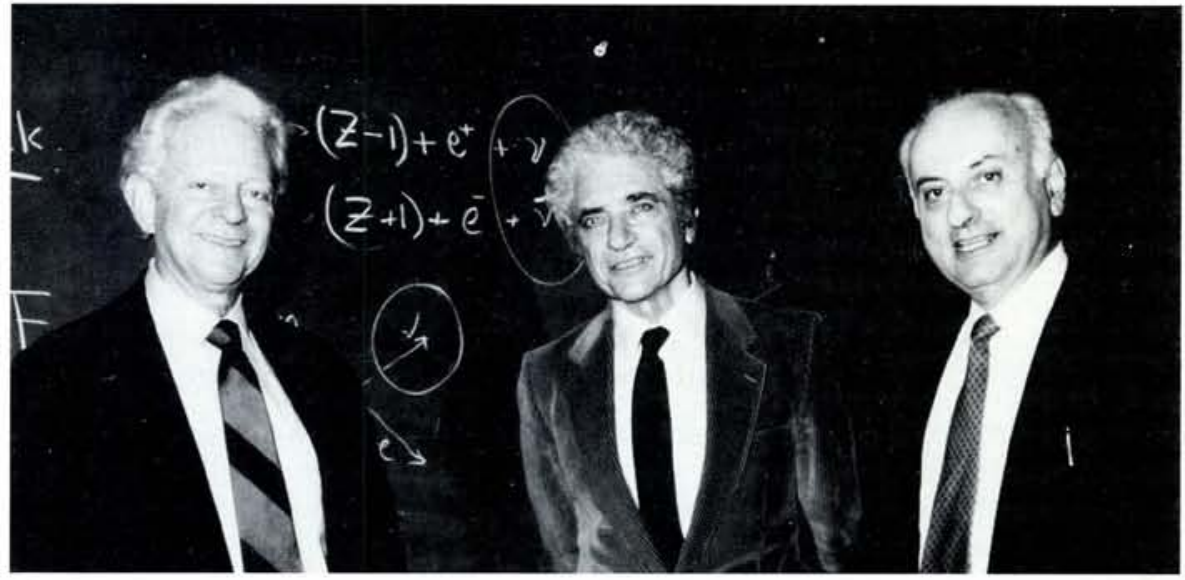

Fig. 1 - A recent photograph taken at CERN of Leon Lederman (left), Jack Steinberger (centre) and Melvin Schwartz.

the muon not decay into $\mathrm{e}+\gamma$ at the rate predicted if such a non-locality existed?". On this view the muon would virtually dissociate into $W+v$, the charged $W$ would radiate a $\gamma$ and $W+v$ would recombine to an electron. What was wrong with this picture? The nonlocality looked like a must to avoid the unitarity catastrophe. Why not postulate two neutrinos, one associated with the electron family and the other with the muon family. This would explain the apparent absence of the decay $\mu \rightarrow \mathrm{e}+\gamma$.

These were exciting and most fundamental questions. They were investigated, independently, by Bruno Pontecorvo [7] and by Melvin Schwartz [8]. Mel Schwartz has related his own discovery story [9]. He had the idea during the night following a coffee break discussion at the Pupin Laboratory of Columbia University in New York in November 1959 of using high energy neutrinos to probe the weak interaction at high energy, T.D. Lee had asked the question. This was the answer: use high energy neutrino beams. Mel Schwartz also gave the method of making such a beam. Produce high energy pions in proton collisions with a target and let them decay. Depending on the decay angle, the neutrinos would have energies up to $43 \%$ of the pion energy. At the new strong focussing proton synchrotrons at CERN (26 GeV) and at Brookhaven National Laboratory (30 GeV), then under construction, muon-neutrino energies bet- ween 1 and $2 \mathrm{GeV}$ should be achievable. Would these synchrotrons though, deliver enough neutrinos? According to their specifications they should accelerate $10^{11}$ protons per second, an unprecedented number, yet it would still require a detector of 10 tons weight to detect one neutrino reaction per day.

The first question was whether the neutrinos from pion decay would be identical with those originating in $\beta$ decay and produce equal numbers of electrons and muons in their capture reactions, or would they be different and produce only muons. The detector would have to be shielded against the enormous flux of pions and muons which would otherwise mask the rare neutrino reactions. Both at CERN and at Brookhaven, beams were designed using internal targets in the vacuum chambers of the synchrotron. A big race between the newly founded European Laboratory (CERN) in Geneva, and Brookhaven started. Who would have the best beam and the best detector? The best detector would be a heavy liquid bubble chamber which could show all details of the reaction, but it was to be another 10 years before Gargamelle, a 10 ton bubble chamber could be built for CERN. Meanwhile, a 1 ton bubble chamber was set up at CERN by Bernardini and Steinberger and a liquid scintillator detector combined with a magnetic cloud chamber by a group headed by Helmuth Faissner. In Brookhaven, 
Gaillard, Lederman and Schwartz made a lucky choice. They adopted the spark chamber which had just been successfully used in an experiment at Berkeley by J. Cronin. The plates could be made massive and it had the required space resolution for discriminating between muons and electron cascade showers. In the Spring of 1961, preparations for the CERN experiment had to be interrupted. Because of the shorter straight sections between the magnets of the synchrotron the neutrino flux was just too low; it had been overestimated.

Jack Steinberger returned to Columbia University and gave up his bubble chamber plans, recognizing the decisive advantage of the longer straight sections of the Brookhaven AGS. A steel shielding wall of $13 \mathrm{~m}$ thickness was built, but tests showed excessive leakage and it turned out to be necessary to reduce the synchrotron energy to $15 \mathrm{GeV}$. A team of seven physicists, considered to be a large group at that time, performed the experiment in 1962 [10], (G. Danby, J.M. Gaillard, K. Goulianos, L.M. Lederman, N. Mistry, M. Schwartz and J. Steinberger). In 800 hours of beam time, unprecedented by any other high energy experiment, it was demonstrated that neutrinos from pion decay produce only muons. Today we know of three lepton families, $\left(e, v_{e}\right),\left(\mu, v_{\mu}\right)$ and $\left(\tau, v_{\tau}\right)$. The notion of fermion families was introduced when the existence of the muon-neutrino was uncovered.

At CERN the sudden need to interrupt the first neutrino experiment led to an advance in technique. The invention of the magnetic horn by Simon van der Meer [11] together with the ejection of the proton beam from the synchrotron onto an external target gave an increase in the neutrino flux of a factor of 10. An enlarged heavy liquid bubble chamber and a spark chamber set-up recorded a few thousand events [12]. The two-neutrino result was confirmed. Using the magnetic horn with opposite polarities, positive and negative pion beams were focussed. They produced neutrinos and antineutrinos, respectively. Measurement of the electric charge of the muons produced by their interaction gave evidence that the lepton number was conserved; neutrinos produce negative muons and antineutrinos produce positive muons. The intermediate boson $\mathrm{W}$ was not found; however, a lower limit to its mass was set by the CERN experiment: If the $W$ exists as a free particle its mass must be larger than $1.8 \mathrm{GeV}$.

From here on the idea of $\mathrm{B}$. Pontecorvo and M. Schwartz to study the weak interaction by using high energy neutri-



Fig. 2 - Schwartz in 1963 with his spark chamber (photo Life Magazine).

nos as probes produced a rich harvest. The prediction based on the theory of weak interactions that the cross-section for neutrino interactions would increase by five orders of magnitude from the reactor neutrino energies used by Cowan and Reines to $1 \mathrm{GeV}$ was dramatically confirmed. In inelastic reactions above $1 \mathrm{GeV}$ the cross-section was found to increase linearly with energy [12]. This unexpected behaviour was later on attributed to a new elementary process, the deep inelastic scattering of neutrinos on quarks. $A$ jet of hadrons results from the fragmentation of the quarks because of their confinement. Comparing results of deep inelastic muon and neutrino scattering, the CDHS Collaboration [13] found a ratio of $5 / 18$ for the structure functions, evidence for the alleged electric charge of quarks of $-1 / 3$ and $2 / 3$.

In December 1972 another dramatic discovery was made using the intense CERN neutrino beam and the gigantic heavy liquid chamber Gargamelle conceived and built by André Lagarrigue. An event in which an electron was recoiling from the interaction with an antimuonneutrino was discovered by the group of Helmuth Faissner in Aachen [14]. This is not the place to tell the story of the neutral weak current interaction, but based on this discovery, a more complete theory of the weak interaction was built by S. Glashow, A. Salam and S. Weinberg [15]. A precise determination of the weak mixing angle, defining the ratio of the weak and the electric coupling constant, was derived from deep inelastic neutral current scattering of neutrinos on isoscalar nuclear targets [16]. The result defined a new, true Fermi energy scale and led to the prediction of the masses of the $W$ and $Z$ bosons which were discovered with these masses at CERN in 1983 [17].

The award of the 1988 Nobel Prize for Physics to Leon Lederman, Mel Schwartz and Jack Steinberger for the first high energy neutrino experiment and the discovery of a second neutrino family will probably not be the last chapter in neutrino physics. We are lacking direct detection of the third neutrino $\left(v_{\tau}\right)$, we are ignorant about the questions of neutrino mass and the level at which lepton number conservation will fail, and whether neutrinos are Dirac or Majorana particles.

\section{REFERENCES}

[1] Wolfgang Pauli, Collected papers, Vol. II (Interscience, N.Y.) p. 1313.

[2] Chadwick J., Z.f. Physik 31 (1914) 210; Ellis C.D., Wooster W.A., Proc. Roy. Soc. (London) A117 (1927) 109; Meitner L., Orthmann W., Z.f. Physik 60 (1930) 143.

[3] Fermi E., Z.f. Physik 88 (1934) 161.

[4] Cowan C.L. jr. and Reines F., Phys. Rev. 107 (1957) 528.

[5] Feynman R. and Gell-Mann M.

[6] Feinberg A., Phys. Rev. 110 (1958) 1482. [7] Portecorvo B., Proc. IXth Int. Conf. High Energy Physics Vol. 1, p. 233, Kiev 1959 and Soviet Physics JETP 37 (1959) 1236.

[8] Schwartz M., Phys. Rev. Lett. 4 (1960) 306.

[9] Schwartz M., Adventures in Experimental Physics, Ed. B. Maglich, 1972, p. 82.

[10] Dany G., Gaillard J.M., Goulianos K., Lederman L.M., Mistry N., Schwartz M. and Steinberger J., Phys. Rev. Lett. 9 (1962) 36. [11] Van der Meer S., CERN 61-7 (1961).

[12] Bienlein J.K. et al., Phys. Lett. 13 (1964) 80; Bernardini G. et al., Phys. Lett. 13 (1964) 86; Bernardini G. et al., Nuovo Cim. 38 (1966) 608

[13] Abramowicz H. et al., Z.f. Physik C17 (1983) 237

[14] Hasert F.J. et al., Phys. Lett. B 46 (1973) 121.

[15] Glashow S.L., Nucl. Phys. 22 (1961) 579; Salam A. and Ward J., Phys. Lett. 13 (1963) 168; Weinberg S., Phys. Rev. Lett. 19 (1967) 1264.

[16] Allaby J. et al., CHARM Coll., Phys. Lett. B 177 (1986) 446; Abramowicz H. et al., CDHS Coll., Phys. Rev. Lett. 57 (1986) 298. [17] Arnison G. et al., UAI Coll., Phys. Lett. B 122 (1983) 103; and B 126 (1983) 398.

Banner M. et al., UA2 Coll., Phys. Lett. B 122 (1983) 476; and B 129 (1983) 130. 